\title{
Immunohistochemical Detection of Alkaline Phosphatase in Formalin- Fixed and Paraffin-Embedded Rat Organs by Means of Avidin-Biotin Peroxidase Complex Method
}

\author{
By \\ Kohsuke CHIDA \\ Department of Pathology, Kitasato University School of Hygienic Sciences, Sagamihara, Kanagawa 228, Japan \\ - Received for Publication, November, 4, $1993-$ \\ Key Words: Alkaline Phosphatase, Rat Organ, Formalin Fixation, Paraffin Embedding, Avidin-Biotin Peroxidase

\begin{abstract}
Summary: The author attempted the detection of alkaline phosphatase (ALP) protein in various rat organs, which were fixed with formalin and emdedded in paraffin, by means of the avidin-biotin peroxidase complex (ABC) method. Reaction products were detected after a usual incubation time of $30 \mathrm{~min}$ at room temperature in anti-ALP antibody. Positive reactions were observed in the kidney and liver but not in the small intestine, and this indicates that the antibody used in the present study reacts only with the tissue-nonspecific type of ALP.
\end{abstract} Complex.

Human ALP isozymes are divided into at least three types: tissue-nonspecific type (liver/bone/ kidney/first-trimester placenta), intestinal type and term-placental type (Sussman et al., 1984; Komoda et al., 1985). In rats, ALP isozymes are divided into the nonspecific type and intestinal type, because the placental type of ALP is the same as its nonspecific type (Goldstein et al., 1980). Although there are some reports on the immunohistochemical localization of ALP in rats, only cryostat sections were used in these experiments (Aihara et al., 1982; Hasegawa et al., 1987; Chida, 1993a). Recently, the author detected ALP protein in rat liver by means of immunohistochemical techniques in ordinary histological specimens which were fixed with formalin and embdded in paraffin (Chida, 1993b). However, the specimens required incubation with anti-ALP antibody for a longer period of time than usual incubation time. Since the ABC method is superior in terms of antigen detection sensitivity (Hsu et al., 1981), it is very often used for immunohistochemical staining on paraffin sections. In the present study, the author attempted to detect localization of ALP in various rat organs, which were fixed with formalin and embedded in paraffin, by means of the $A B C$ method.

\section{Materials and Methods}

\section{Fixation and Embedding}

Various organs (liver, kidney, small intestine, pancreas, heart, stomach, spleen, brain, lung, ovary, uterus, testis, thyroid gland, salivary gland, adrenal gland, skin, prostate, seminal vesicle and thymus) were excised from Wistar-strain rats, weighing $180-200 \mathrm{~g}$. The tissue fragments were fixed in $10 \%$ formalin for $24 \mathrm{hr}$ at room temperature. After washing with tap water overnight, the tissue fragments were embedded in paraffin following the conventional procedure.

\section{Immunohistochemical staining}

Paraffin sections were cut into 5- $\mu$ m-thick slices. After paraffin was removed using xylene, the sections were incubated in $0.3 \%$ hydrogen peroxide in absolute methanol for $30 \mathrm{~min}$ at room temperature in order to inactivate endogeneous peroxidase. The sections were then washed with $0.01 \mathrm{M}$ phosphate

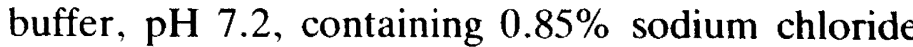
(PBS) and $10 \%$ normal horse serum was placed on the sections for $20 \mathrm{~min}$ at room temperature. After washing with PBS, anti-rat kidney ALP rabbit antibody (Chida, 1993a) diluted 50-fold with PBS was applied to the sections and left for $30 \mathrm{~min}$ at room termperature. Thereafter, the sections were washed with PBS, and incubated with an avidinbiotin-peroxidase complex $(\mathrm{ABC})$ kit (Vectastain ABS kit; Vector Laboratories, Burlingame, CA). 
In order to detect peroxidase activity, the sections were immersed in diaminobenzidine (DAB) solution (Graham and Karnovsky, 1966) for $5 \mathrm{~min}$ at room temperature. Afterwards, the sections were washed with distilled water and mounted with glycerin jelly after the nuclei were stained with $1 \%$ methylgreen.

\section{Results}

Reaction products were detected after a usual incubation time of $30 \mathrm{~min}$ at room temperature in anti-ALP antiobody. In the kidney and liver, positive reactions were observed in the brush borders of the epithelial cells of proximal tubules (Fig. 1), and along the cell borders, i.e., bile canaliculi, of adjacent hepatocytes (Fig. 2), respectively. In the pancreas, positive reactions were present in the apical surface of centroacinar cells and of the epithelial cells of ducts (Fig. 3). On the other hand, in the small intestine and other organs, no reaction was observed (Fig. 4).

\section{Discussion}

Immunohistochemical staining of ALP in ordinary histological specimens, which were fixed with formalin and embedded in paraffin, is useful for easy detection of the localization of enzyme proteins under various experimental conditions. However, in the previous paper (Chida, 1993b), for immunohistochemical detection of ALP in ordinary histological specimens, the specimens required incubation with anti-ALP antibody for a longer period of time, i.e., $3 \mathrm{hr}$ at room temperature, as compared with ordinary incubation time. The $\mathrm{ABC}$ method is superior in terms of antigen detection sensitivity (Hsu et al., 1981). In the present study, the author detected positive reactions after a usual incubation time of $30 \mathrm{~min}$ at room temperature in anti-ALP antibody by means of the ABC method. Accordingly, the author found that we can easily detect the localization of ALP under various experimental conditions using ordinary histological specimens and the $\mathrm{ABC}$ method. In addition, positive reactions were detected in the kidney and liver but not in the small intestine, and this indicates that the antibody used in the present study reacts only with the tissue-nonspecific type of ALP.

\section{References}

1) Aihara, K., Nagura, H., Yoshimura, S., Hasegawa, H. and Watanabe, K.: Immunohistochemical and enzymecytochemical localization of intestinal alkaline phosphatase of rats. Acta Histochem. Cytochem 1982;15:163-175.

2) Chida, K.: Immunohistochemical localization of alkaline phosphatase in rat liver. Acta Histochem. Cytochem 1993a; 26:239-244.

3) Chida, K.: Immunohistochemical detection of alkaline phosphatase in formalin-fixed and paraffin-embedded rat liver. Okajimas Folia Anat. Jpn 1993b;70: in press.

4) Goldstein, D. J., Roger, C. E. and Harris, H.: Expression of alkaline phosphatase loci in mammalian tissues. Proc. Natl. Acad. Sci. U.S.A. 1980;77:2857-2860.

5) Graham, R. C. and Karnovsky, M. J.: The early stage of absorption of injected horseradish peroxidase in the proximal tubules of mouse kidney; ultrastructural cytochemistry by a new technique. J. Histochem. Cytochem 1966;14:291-302.

6) Hasegawa, H., Watanabe, K., Nakamura, T. and Nagura, H.: Immunohistochemical localization of alkaline phosphatase in absorptive cells of rat small intestine after colchicine treatment. Cell Tissue Res 1987;250:521-529.

7) Hsu, S. -M., Raine, L. and Fanger, H.: A comparative study of the peroxidase-antiperoxidase method for studying peptide hormones with radioimmunoassay antibodies. Am. J Clin. Pathol 1981;75:734-738.

8) Komoda, T., Koyama, I. and Sakagishi, Y.: Focusing into tumor and/or fetal-associated alkaline phosphatase and its translocation mechanism to blood stream. Protein, nucleic acid, and enzymes 1985;30:1408-1420. (in Japanese).

9) Sussman, H. H.: Structural analysis of human alkaline phosphatase. In "Human Alkaline Phosphatases", Eds. by T. Stigbrand and W. H. Fishman 1984; 87-103, Alan R. Liss, New York.

\section{Explanation of Figures}

\section{Plate I}

Fig. 1. Localization of alkaline phosphatase in rat kidney. Positive reactions are observed in the brush borders of the epithelial cells of proximal tubules. $\times 230$.

Fig. 2. Localization of alkaline phosphatase in rat liver. Positive reactions are observed along cell borders, i.e., bile canaliculi, of adjacent hepatocytes and in the apical surface of the epithelial cells of bile ducts. $\times 450$. 

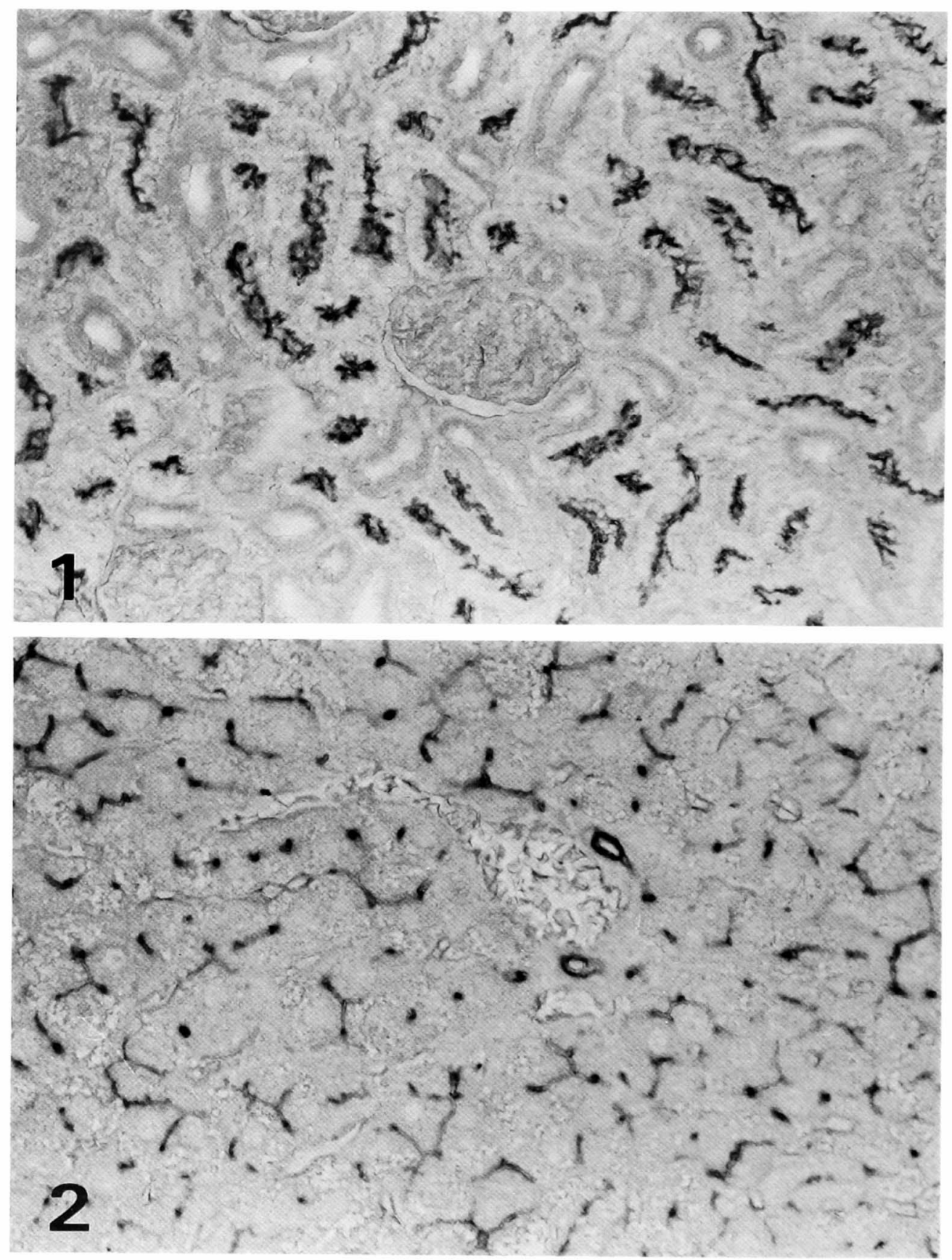


\section{Plate II}

Fig. 3. Localization of alkaline phosphatase in rat pancreas. Positive reactions are observed in the surface of centroacinar cells and of the epithelial cells of ducts. $\times 450$.

Fig. 4. Immunohistochemical staining of alkaline phosphatase in rat small intestine. No reaction is observed. $\times 230$. 

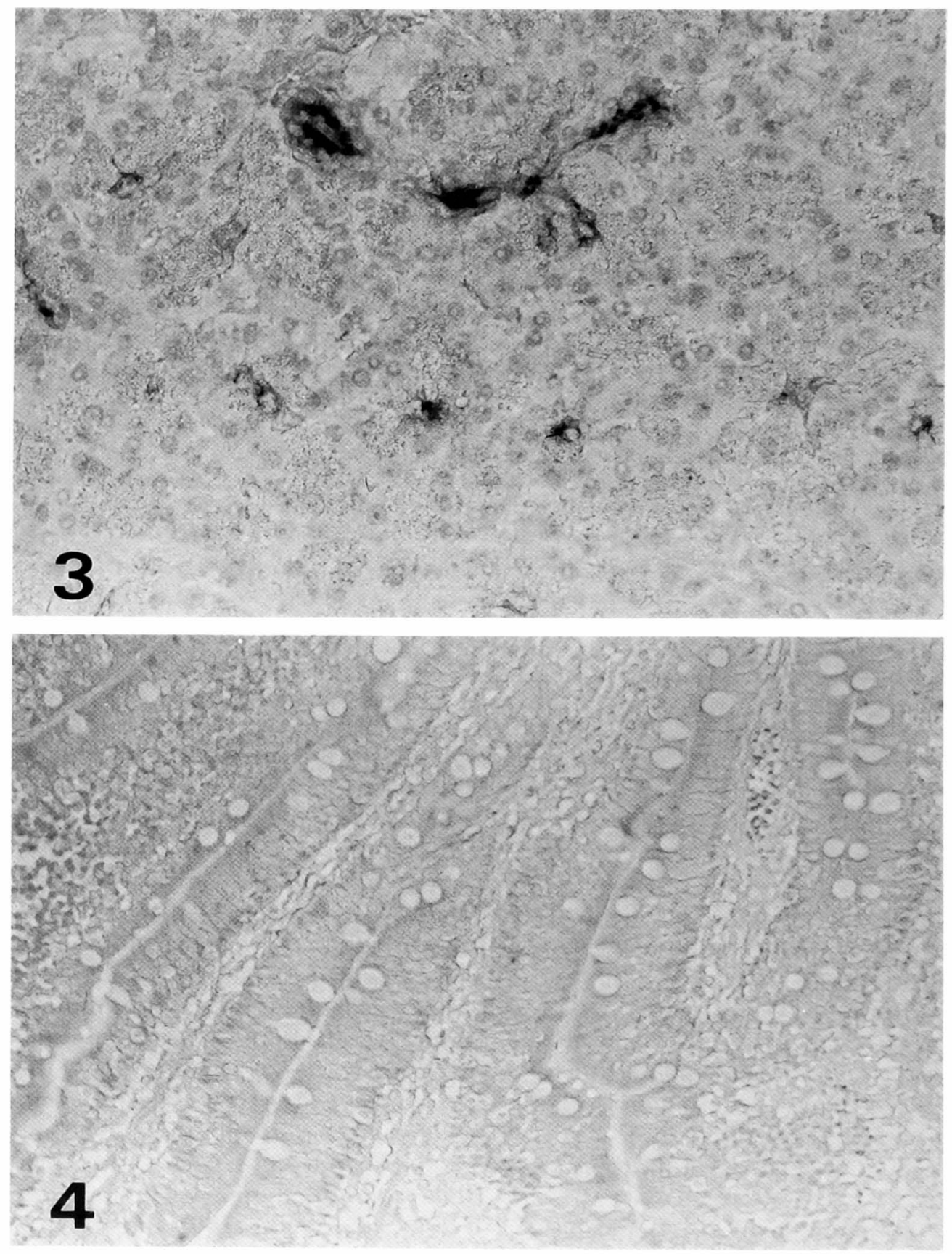